\title{
ACOMPANHAMENTO DO TRATAMENTO QUIMIOTERÁPICO DO OSTEOSSARCOMA POR IMAGEM INFRAVERMELHA
}

\author{
Michel Freire de Araújoㅁ, Marcos Leal Brioschi²
}

1. Coordenador Médico da Oncologia Ortopédica da Liga Contra o Câncer, Natal, Brasil.

2. Professor Coordenador da Pós-graduação em Termografia e Termologia Médica da FMUSP, São

Paulo, Brasil.

\section{RESUMO}

Os tumores ósseos primários malignos são doenças raras, sendo o mais frequente o osteossarcoma. O diagnóstico é feito por exames de imagem e anatomopatológico, sendo tratado em três etapas (quimioterapia pré-cirúrgica, cirurgia e quimioterapia pós-cirúrgica). 0 mais relevante para o prognóstico a resposta tumoral à quimioterapia. O objetivo deste estudo foi avaliar a evolução do tratamento quimioterápico de pacientes com tumor ósseo por meio das imagens térmicas. Foi realizado um estudo prospectivo observacional com 04 pacientes (3 do gênero masculino e 1 do gênero feminino) com tumor ósseo maligno primário (Osteossarcoma). Os resultados mostram que o comportamento térmico coincidiu com a melhora clínica dos pacientes no decorrer do tratamento, com relação a dor e calor local. Pode-se concluir que a termografia por infravermelho é um exame de imagem funcional indolor, sem radiação e sem riscos que pode ser utilizado como mais uma ferramenta no acompanhamento da resposta tumoral ao tratamento quimioterápico.

Palavras-Chave: Termografia, Infravermelho, Osteossarcoma, Tumor, Osso.

\section{ABSTRACT}

Primary malignant bone tumors are rare diseases, the most frequent being osteosarcoma. The diagnosis is made by imaging and pathological examinations, and is treated in three stages (pre-surgical chemotherapy, surgery, and post-surgical chemotherapy). The most relevant for prognosis is the tumor response to chemotherapy. The aim of this study was to evaluate the evolution of chemotherapy treatment of patients with bone tumor by means of thermal images. A prospective observational study was performed with 04 patients ( 3 males and 1 female) with primary malignant bone tumor (Osteosarcoma). The results shows that the thermal behavior coincided with the clinical improvement of patients during treatment, with regard to pain and local heat. It can be concluded that infrared thermography is a painless, radiation-free and risk-free functional imaging test that can be used as an-other tool in monitoring tumor response to chemotherapy treatment.

Key Words: Thermography, Infrared, Osteosarcoma, Tumor, Bone.

DOI: http://dx.doi.org/10.18073/pajmt.2020.7.002 


\section{INTRODUÇÃO}

Os tumores ósseos são uma doença rara, representam apenas $2 \%$ dos tumores que podem acometer um ser humano. 0 diagnóstico precoce e o imediato encaminhamento do paciente para um Serviço de referência são fundamentais para o resultado do tratamento ${ }^{1}$. A dor é o sintoma principal, e na maioria das vezes o primeiro a surgir, apenas com a evolução do quadro é que aparece a tumoração.

Um dos motivos para o elevado tempo entre o diagnóstico e o início do tratamento é o fato dos médicos não pensarem em tumores ósseos como diagnóstico diferencial ${ }^{1}$.

Uma vez frente a um quadro suspeito de tumor ósseo, é mandatório prosseguir com exames de estadiamento que incluem radiografias, tomografias, cintilografia óssea, ressonância magnética, exames laboratoriais, e a biópsia para o exame anatomopatológico e estudo imuno-histoquímico².

O osteossarcoma é o tumor ósseo maligno primário mais frequente da infância, principalmente na segunda década de vida ${ }^{3}$.

Antes de 1970 o tratamento do osteossarcoma era exclusivamente cirúrgico, através da amputação do membro, o prognóstico era péssimo, com sobrevida de $20 \%$ em 05 anos. Após 1970, iniciou o uso de quimioterápicos no tratamento e isso modificou totalmente a sobrevida, passando para $60 \%$ em 05 anos. Na década de 80 se intensificou os estudos com os quimioterápicos, com drogas mais modernas e protocolos mais eficientes, chegando, em alguns estudos, a mostrar uma sobrevida de $80 \%$ em 05 anos $^{2}$.

Com a melhora da sobrevida, surgiu uma maior exigência na qualidade de vida dos pacientes, principalmente com relação a funcionalidade do membro.
Desta forma, várias técnicas surgiram com objetivo de salvamento do membro ("Limb Salvage"). Vários modelos de endo próteses não-convencionais para reconstruir as grandes ressecções ósseas, cirurgias mais biológicas com uso de aloenxerto, e de enxerto autógeno congelado, "Frozen Surgery"4,5. Mesmo quando a amputação é a única técnica cirúrgica possível, houve grande desenvolvimento tecnológico na performance das próteses. Associado a todos esses esforços, se desenvolveu conjuntamente métodos e técnicas de reabilitação em busca de tornar o paciente mais produtivo e independente ${ }^{6}$.

Atualmente o tratamento consiste em quimioterapia pré-operatória, num protocolo de 10 ciclos, logo em seguida é realizado o tratamento cirúrgico, com a ressecção do tumor com margem oncológica, e em seguida quimioterapia pós-operatória, com mais 10 ciclos $^{2}$.

O principal fator prognóstico desses pacientes com osteossarcoma é a resposta tumoral ao tratamento quimioterápico. Essa avaliação é feita através da melhora dos sintomas (principalmente a dor), exames de imagens (para acompanhar a redução do volume tumoral), e a quantificação do grau de necrose tumoral (Classificação de Huvos) que é feita pelo exame anatomopatológico da peça cirúrgica7.

O exame de imagem infravermelha é um método diagnóstico funcional de imagem, realizado através de uma câmera especial que capta radiação infravermelha longa emitida pelo corpo humano (6 a $15 \mu \mathrm{m})$, proporcionando uma imagem da distribuição térmica da superfície cutânea ${ }^{8}$.

O estímulo doloroso nociceptivo e neuropático ativam o sistema neurovegetativo simpático que controla a atividade micro circulatória cutânea, influ- 
enciando a temperatura da pele, juntamente com o calor produzido em tecidos mais profundos ${ }^{8}$. Além disso, o aumento da atividade metabólica tumoral provoca uma neovascularização periférica, que também pode ser analisada pela imagem in-fravermelha ${ }^{9,10}$.

Dessa forma, o objetivo deste estudo foi avaliar a evolução do tratamento quimioterápico de pacientes com tumor ósseo por meio das imagens térmicas.

\section{METODOLOGIA}

Realizado estudo prospectivo observacional transversal com pacientes com tumor ósseo maligno primário (Osteossarcoma). A população estudada consta de 6 indivíduos, sendo 5 do gênero masculino e 1 do gênero feminino. Sendo que dois pacientes do gênero masculino foram excluídos, pois o diagnóstico anatomopatológico foi incompatível com Osteossarcoma (Condrossarcoma e Sarcoma de Ewing). Então, participaram do estudo 04 paciente (3 do gênero masculino e 1 do gênero feminino) com quadro de dor e aumento de volume na região do joelho. $A$ idade variou de 08 a 20 anos.

Foi seguido o protocolo padrão de diagnóstico e estadiamento da instituição (LIGA Contra o Câncer) para casos suspeitos de tumor ósseo maligno, através de exames de imagem (Radiografia e Ressonância Nuclear Magnética da região tumoral, Cintilografia Óssea, Tomografia Computadorizada de tórax e abdome) exames laboratoriais. Neste momento foi acrescentado imagem termográfica de infravermelho do segmento acometido e seu correspondente anatômico contralateral. Só então foi realizada biópsia percutânea para coleta de material tumoral, exame anatomopatológico e imuno-histoquímica.
Após concluído o diagnóstico, iniciou-se o tratamento quimioterápico pré-operatório por 10 semanas, sendo colhido imagens térmicas de infravermelho na $4^{a}$ semana, $7^{a}$ semana e $10^{a}$ semana.

Como a lesão tumoral em todos os pacientes estava localizada no fêmur distal, bem próxima ao joelho, eles apresentavam, no decorrer do tratamento quimioterápico deformidade em flexo de joelho, o que gerou uma dificuldade no posicionamento (incapacidade em estender o joelho e deixar o pé plantígrado) adequado para coleta das imagens.

Antes do exame termográfico, os indivíduos permaneceram em repouso durante 15 minutos em ambiente termicamente controlado com climatizador de $a r$, em temperatura entre $22-23^{\circ} \mathrm{C}$, com velocidade do ar menor que $0,2 \mathrm{~m} / \mathrm{s}$, dentro de um consultório médico, para fins de aclimatação ${ }^{11}$. Os pacientes foram submetidos a exame termográfico para registro da temperatura do segmento acometido e seu correspondente anatômico contralateral. As imagens foram coletadas com o paciente em posição frontal em pé, com câmera termográfica FLIR modelo T530.

Foi confeccionado um padrão para avaliação dos pontos de análise, através do software WORD FLIR TOOLS +, sendo divididos de acordo com a área correspondente (Tabela 1).

Em cada paciente foi realizado imagem termográfica de infravermelho da região anterior dos membros inferiores em 4 momentos (pré-quimioterapia, no $4^{0}$ ciclo, $7^{\circ}$ ciclo e $10^{\circ}$ ciclo, cada ciclo equivale a uma semana). Sendo mensurada a temperatura média de três regiões de interesse (ROI), na área da tumoração (ROI1), na equivalente contralateral (ROI2), e na área distal a tumora- 
ção (ROI3), além disso foi medido a diferença entre a média das ROls (Figura1).

Tabela 1. Imagem representação das regiões tumorais e sua região correspondente contralateral.

\begin{tabular}{|c|c|}
\hline Área & Local \\
\hline ROI1 & Região tumoral \\
\hline ROI2 & Contralateral a ROI1 \\
\hline ROI3 & Distal a ROI1 \\
\hline
\end{tabular}

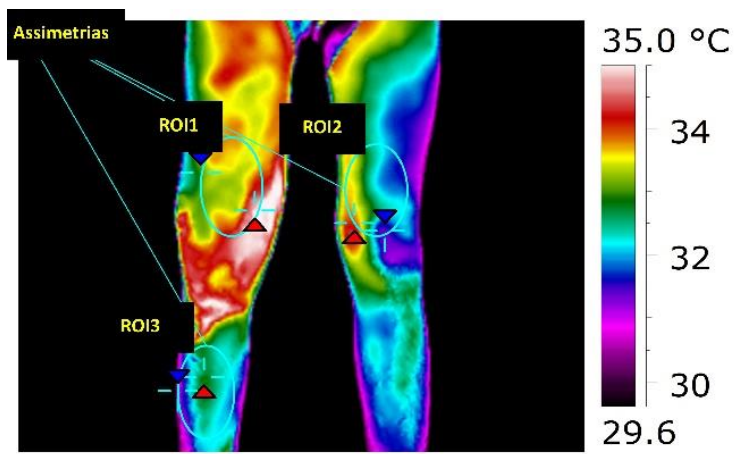

Figura 1. Ilustração da seleção dos ROIs.

\section{RESULTADOS}

Entre o período de julho de 2020 a janeiro de 2021 foram avaliados 04 pacientes. Destes, 03 pacientes eram do gênero masculino, representando $75 \%$ da amostra, enquanto 01 era do gênero feminino (25\%). Dentre os pacientes, todos tinham tumor ósseo na região do joelho (fêmur distal).

Tabela 2. Distribuição dos pacientes com osteossarcoma de joelho, por sexo e idade.

\begin{tabular}{|c|c|c|}
\hline Paciente & Sexo & Idade \\
\hline 1 & Masculino & 12 \\
\hline 2 & Masculino & 20 \\
\hline 3 & Masculino & 8 \\
\hline 4 & feminino & 12 \\
\hline
\end{tabular}

Os resultados térmicos encontrados em cada paciente estão apresentados na Tabela 3.
Tabela 3. Temperatura das Regiões de Interesse (ROIs) de pacientes com osteossarcoma de joelho ao longo do período de tratamento $\left({ }^{\circ} \mathrm{C}\right)$

\begin{tabular}{|c|c|c|c|c|}
\hline ROI & Momento & $\begin{array}{l}\text { Média } \\
\left({ }^{\circ} \mathrm{C}\right)\end{array}$ & $\begin{array}{c}\text { Mediana } \\
\left({ }^{\circ} \mathrm{C}\right)\end{array}$ & $\begin{array}{l}\text { DP } \\
\left({ }^{\circ} \mathrm{C}\right)\end{array}$ \\
\hline \multirow{4}{*}{ 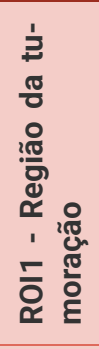 } & $\begin{array}{l}\text { Pré Qui- } \\
\text { miotera- } \\
\text { pia }\end{array}$ & 32,05 & 32,15 & 1,60 \\
\hline & $\begin{array}{l}\text { Após } 4 \text { Ci- } \\
\text { clos }\end{array}$ & 33,50 & 33,35 & 1,85 \\
\hline & $\begin{array}{l}\text { Após } 7 \text { Ci- } \\
\text { clos }\end{array}$ & 31,88 & 31,50 & 1,76 \\
\hline & $\begin{array}{l}\text { Após } 10 \\
\text { Ciclos }\end{array}$ & 31,78 & 31,15 & 2,46 \\
\hline \multirow{4}{*}{ 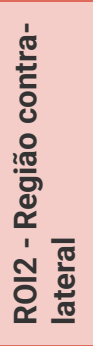 } & $\begin{array}{l}\text { Pré Qui- } \\
\text { miotera- } \\
\text { pia }\end{array}$ & 30,43 & 31,10 & 2,40 \\
\hline & $\begin{array}{l}\text { Após } 4 \text { Ci- } \\
\text { clos }\end{array}$ & 32,18 & 32,20 & 2,22 \\
\hline & $\begin{array}{l}\text { Após } 7 \text { Ci- } \\
\text { clos }\end{array}$ & 31,73 & 31,35 & 1,41 \\
\hline & $\begin{array}{l}\text { Após } 10 \\
\text { Ciclos }\end{array}$ & 32,13 & 31,75 & 2,00 \\
\hline \multirow{4}{*}{ 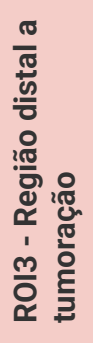 } & $\begin{array}{l}\text { Pré Qui- } \\
\text { miotera- } \\
\text { pia }\end{array}$ & 30,50 & 30,85 & 1,78 \\
\hline & $\begin{array}{l}\text { Após } 4 \text { Ci- } \\
\text { clos }\end{array}$ & 31,78 & 31,85 & 1,52 \\
\hline & $\begin{array}{l}\text { Após } 7 \mathrm{Ci}- \\
\text { clos }\end{array}$ & 30,88 & 30,90 & 1,02 \\
\hline & $\begin{array}{l}\text { Após } 10 \\
\text { Ciclos }\end{array}$ & 31,53 & 30,90 & 1,83 \\
\hline \multirow{4}{*}{ 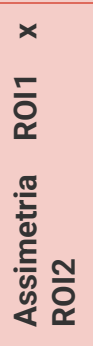 } & $\begin{array}{l}\text { Pré Qui- } \\
\text { miotera- } \\
\text { pia }\end{array}$ & 1,63 & 1,40 & 1,24 \\
\hline & $\begin{array}{l}\text { Após } 4 \text { Ci- } \\
\text { clos }\end{array}$ & 1,33 & 1,50 & 1,46 \\
\hline & $\begin{array}{l}\text { Após } 7 \mathrm{Ci}- \\
\text { clos }\end{array}$ & 0,15 & 0,15 & 0,71 \\
\hline & $\begin{array}{l}\text { Após } 10 \\
\text { Ciclos }\end{array}$ & $-0,35$ & $-0,10$ & 1,17 \\
\hline \multirow{4}{*}{ 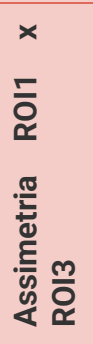 } & $\begin{array}{l}\text { Pré Qui- } \\
\text { miotera- } \\
\text { pia }\end{array}$ & 1,55 & 1,60 & 0,60 \\
\hline & $\begin{array}{l}\text { Após } 4 \text { Ci- } \\
\text { clos }\end{array}$ & 1,73 & 1,80 & 0,67 \\
\hline & $\begin{array}{l}\text { Após } 7 \mathrm{Ci}- \\
\text { clos }\end{array}$ & 1,00 & 1,00 & 1,14 \\
\hline & $\begin{array}{l}\text { Após } 10 \\
\text { Ciclos }\end{array}$ & 0,25 & 0,25 & 0,93 \\
\hline
\end{tabular}

Na Figura 2, pode-se verificar a redução das assimetrias entre as ROI1 $x$ ROI2 ao longo do tratamento, e a Figura 3 mostra e evolução das assimetrias entre as ROI1 x ROI3. 


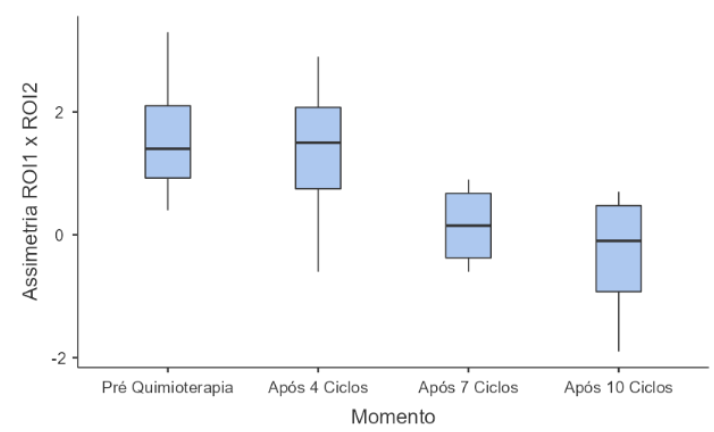

Figura 2. Assimetria média entre as R01xROI2 (Todos os pacientes).

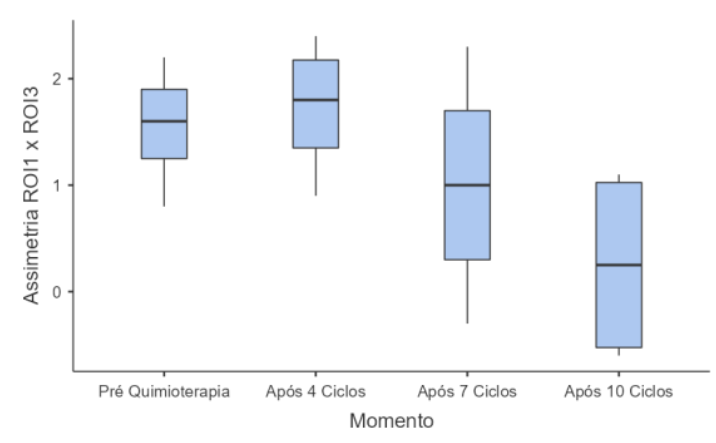

Figura 3. Assimetria média entre as R01xROI3 (Todos os pacientes).

\section{DISCUSSÃO}

O presente estudo avaliou a temperatura dos membros inferiores em pacientes com diagnóstico, confirmado por exame anatomopatológico, de Osteossarcoma do fêmur distal. Trata-se de um trabalho inédito em literatura brasileira e pouco descrito em estudos internacionais.

A pele apresenta uma rede de vasos acompanhados por fibras nervosas densas, atuam na termorregulação, controlando o fluxo sanguíneo na derme. O sistema é anatômico e fisiologicamente simétrico em relação a ambos os lados do corpo. Por este motivo, alterações na simetria da temperatura do corpo tem interessado muito a área médica. 0 recente desenvolvimento de dispositivos sofisticados de termografia pode nos fornece um mapa da tem- peratura do corpo, indicando - em casos de assimetria térmica - uma possível alteração patológica ${ }^{12,13}$.

A termografia útil como método de diagnóstico funcional não ionizante, não invasivo e sem riscos. A análise da simetria térmica do corpo em pessoas hígidos mostram que a temperatura absoluta corporal entre regiões homólogas é extremamente confiável. $\mathrm{E}$ a variação de temperatura contralateral obtidos para os sujeitos normais pode ser utilizada como um padrão de referência para a comparação da mesma variação em sujeitos com alguma suspeita clínica. Então, a avaliação das diferenças entre os valores padronizados nos estudos $^{14-16}$ permite a suspeita de doenças neurológicas, musculoesqueléticas, vasculares, metabólicas.

Estudo preliminar ${ }^{17}$ indica que a termografia é útil na avaliação de pacientes com osteossarcoma. Pode ser empregado para determinar a extensão local e vascularização do tumor primário, recorrência e metástases. O termograma pode apresentar achados não imediatamente detectáveis pelos métodos convencionais de diagnóstico.

Os tumores ósseos primários malignos são doenças raras, se comparado aos demais tumores, sendo o Osteossarcoma o mais frequente. Tendo como principal sintoma a dor, seguido por calor local e tumoração. Devido a alta taxa metabólica tumoral, surge circulação colateral, e o aumento da temperatura da região ${ }^{17}$. Tanto a dor como o aumento do metabolismo tumoral provocam respostas simpáticas, liberação de neurotransmissores, inflamação neurogênica, aumento do fluxo sanguíneo local. Todos esses estímulos provocam respostas na termorregulação, podendo ser captada por equipamentos de infravermelho ${ }^{18}$.

O tratamento do Osteossarcoma é dividido em três etapas (quimioterapia 
pré-operatória, cirurgia e quimioterapia pós-operatória), sendo que o elemento mais importando para o prognóstico é a resposta tumoral ao tratamento quimioterápico. Durante os ciclos de quimioterapia neoadjuvante, a avaliação da resposta tumoral se dá através da análise clínica, com melhora dos sintomas e redução da tumoração e calor local pelo exame físico. A avaliação radiológica por Ressonância Magnética é feita na fase pré-quimioterapia e novamente, apenas, após o $10^{\circ} \mathrm{CICLO}$, para verificar se o tumor respondeu ao tratamento quimioterápico.

Nas Figuras 2 e 3 observou-se uma tendência de redução da diferença térmica de infravermelho entre a Área acometida e a Área contralateral sadia, e também houve redução da diferença térmica de infravermelho entre a Área acometida e a Área mais distal ipsilateral. Chegando, após o 7 CICLO, a Área tumoral ter uma temperatura de infravermelho média inferior às áreas sadias.

Esse comportamento térmico coincide com a melhora clínica dos pacientes no decorrer do tratamento, com relação a dor e calor local. Com equipamentos de termografia por infravermeIho mais modernos e sensíveis é possível perceber varrições de $0,01-0,06^{\circ} \mathrm{C}$ por $\mathrm{mm}^{2}$ de pele, tornando a termografia um método funcional de fácil execução, muito útil no diagnóstico e acompanhamento da resposta tumoral ao tratamento, podendo ser realizado várias vezes no decorrer do tratamento.

A literatura ainda carece de estudos que relacionam a temperatura da área tumoral com a resposta ao tratamento quimioterápico nos sarcomas. No presente estudo foi possível observar que a curva térmica de infravermelho pode ser um instrumento valioso no acompanhamento da resposta tumoral ao tratamento, nos fornecendo informações quanto a eficácia da terapêutica medicamentosa, e o momento ideal para a etapa cirúrgica.

\section{CONCLUSÃO}

As temperaturas emitidas na região tumoral apresentam assimetria térmica com a região homóloga contralateral e a região distal homolateral. A temperatura da região tumoral se modifica, reduzindo seus valores no decorrer do tratamento quimioterápico, tendendo a atingir suas mínimas por volta do $7^{\circ} \mathrm{ci}$ clo. E coincide com a melhora clínica dos pacientes (dor e calor local).

A quantidade de casos não nos permite ter conclusões mais aprofundadas. Estudos com maior número de pacientes podem nos ajudar a evidenciar se a termografia seria útil na decisão de mudança de esquema quimioterápico, ou do melhor momento para cirurgia.

\section{Agradecimentos}

Ao Professor Manoel Jacobsen Teixeira - Médico Neurocirurgião Professor Titular da Disciplina de Neurocirurgia do Departamento de Neurologia do Hospital das Clínicas da FMUSP

\section{REFERÊNCIAS}

1. Jesus-Garcia, R. Diagnóstico e tratamento de tumores ósseos - 2. ed. - Elsevier, 2013; 363.

2. Ritter, J., \& Bielack, S. S. Osteosarcoma. Annals of oncology, 2010; 21: vii320-vii325.

3. Kager, L., Tamamyan, G., \& Bielack, S. Novel insights and therapeutic interventions for pediatric osteosarcoma. Future Oncology. 2017; 13(4): 357-368.

4. Tsuchiya, H., Nishida, H., Srisawat, P., Shirai, T., Hayashi, K., Takeuchi, A., ... \& Tomita, K. Pedicle frozen autograft reconstruction in malignant bone tumors. Journal of Orthopaedic Science. 2010; 15(3): 340-349. 
5. Shimozaki, S., Yamamoto, N., Shirai, T., Nishida, H., Hayashi, K., Tanzawa, Y., ... \& Tsuchiya, $\mathrm{H}$. Pedicle versus free frozen autograft for reconstruction in malignant bone and soft tissue tumors of the lower extremities. Journal of Orthopaedic Science. 2014; 19(1): 156-163.

6. Grinberg, S. Z., Posta, A., Weber, K. L., \& Wilson, R. J. Limb Salvage and Reconstruction Options in Osteosarcoma. In Current Advances in Osteosarcoma. Springer, Cham. 2020: pp. 13-29.

7. Biazzo, A., \& De Paolis, M. Multidisciplinary approach to osteosarcoma. Acta Orthop Belg. 2016; 82(4): 690-698.

8. Brioschi ML, Yeng, LT, Pastor, EMH, Colman, D, Silva, FMRM, Teixeira, MJ. Documentação da síndrome dolorosa miofascial por imagem infravermelha. Acta Fisiatrica Br. 2007; 14(1): 41-48.

9. Miwa, S., Takeuchi, A., Ikeda, H., Shirai, T., Yamamoto, N., Nishida, H., ... \& Tsuchiya, $H$. Prognostic value of histological response to chemotherapy in osteosarcoma patients receiving tumor-bearing frozen autograft. PLoS One. 2013; 8(8): e71362.

10. Gellermann J, Hildebrandt $B$, Issels $R$, Ganter H, Wlodarczyk W, Budach V, Felix R, Tunn PU, Reichardt P, Wust P. Noninvasive magnetic resonance thermography of soft tissue sarcomas during regional hyperthermia: correlation with response and direct thermometry. Cancer. 2006; 15;107(6):137382.

11. Neves, E. B., Reis, V. M. Fundamentos da termografia para o acompanhamento do treinamento desportivo. Revista Uniandrade. 2014; 15(2): 79-86.

12. Kabet B, Yamamoto V, Yu C, Grundfest W, Gruen JP. Infra-red thermal imaging: a review of the literature and case report. Neuroimage. 2009; 47:154-162.

13. de Meira, L. F., Krueger, E., Neves, E. B., Nohama, P., \& de Souza, M. A. Termografia na área biomédica. Pan American Journal of Medical Thermology. 2014; 1(1): 31-41.

14. Neves, E. B., Vilaça-Alves, J., Rosa, C., \& Reis, $\mathrm{V}$. M. Thermography in neurologic practice. The open neurology journal. 2015; 9: 24-27.

15. Mendes, R., Sousa, N., Almeida, A., VilaçaAlves, J., Reis, V. M., \& Neves, E. B. Thermography: a technique for assessing the risk of developing diabetic foot disorders. Postgraduate medical journal. 2015; 91(1079): 538-538.

16. dos Santos Bunn, P., Miranda, M. E. K., Rodrigues, A. I., de Souza Sodré, R., Neves, E. B., \& da Silva, E. B. Infrared thermography and musculoskeletal injuries: a systematic review with meta-analysis. Infrared Physics \& Technology. 2020: 109: 103435.

17. Farrell C, Wallace JD, Edeiken J. Thermography and oste-osarcoma. Radiology. 1968; 90(4): 792-3.

18. Gautherie, M., Gros, C. M. Breast thermography and cancer risk prediction. Cancer. 1980; 45(1): 51-56. 